\title{
Adult linear IgA bullous dermatosis: report of three cases*
}

\author{
Taila Yuri Siqueira Machado ${ }^{1}$ \\ Tatiana Miyuki Iida ${ }^{1}$
}

\author{
Milvia Maria Simões e Silva Enokihara ${ }^{1,2}$ \\ Adriana Maria Porro ${ }^{1}$
}

DOI: http:/ /dx.doi.org/10.1590/abd1806-4841.20187304

\begin{abstract}
Linear immunoglobulin A bullous dermatosis is a rare autoimmune disease that usually has an excellent prognosis in childhood; however, its control is more difficult in adults. It presents heterogeneous clinical manifestations and is frequently confused with other bullous diseases such as bullous pemphigoid and Duhring's dermatitis herpetiformis. Dermatologists' awareness of this disease contributes to early diagnosis and appropriate treatment. We thus report three cases of linear immunoglobulin A dermatosis in adults.
\end{abstract}

Keywords: Immunoglobulin A; Linear IgA bullous dermatosis; Skin diseases, vesiculobullous

\section{INTRODUCTION}

Described for the first time in the 1970s as a different entity from dermatitis herpetiformis, linear IgA dermatosis is a rare autoimmune bullous disease characterized by subepidermal blisters and linear deposition of IgA in the basement membrane zone, shown on direct immunofluorescence (DIF). ${ }^{1,2}$ Two clinical forms are known: in childhood, with a peak incidence at four to five years of age, with excellent prognosis and rarely persisting beyond puberty, and in adulthood, which occurs after puberty or after 60 to 65 years of age and presents less frequent remission. ${ }^{3}$

Considering the rarity of this disease in adults and the importance of the dermatologist's awareness of it for early diagnosis, we report three cases of linear IgA dermatosis in adults.

\section{CASE REPORTS}

Case 1

Female patient, 44 years, complained of lesions on her skin in the previous 50 days. On examination, she presented vesicles, blisters, and hemorrhagic crusts with an arciform configuration on her neck, trunk, and lower limbs, with no mucosal lesions (Figure 1). She reported no comorbidities or recent use of medications. Anatomopathology (AP) showed subepidermal bullous dermatosis with microabscesses in the papillary dermis, and DIF showed moderate linear IgA fluorescence in the basement membrane zone - findings consistent with linear IgA dermatosis (Figures 2 and 3). Patient was treated with prednisone (maximum dose $60 \mathrm{mg} /$ day, the equivalent of $1 \mathrm{mg} / \mathrm{kg} /$ day) and dapsone (maximum dose 100mg/ day, reduced to $50 \mathrm{mg} /$ day due to hemolysis). Five years after onset of the condition, she presented glaucoma, arterial hypertension, osteoporosis, and Cushingoid facies as side effects of prolonged corticosteroid therapy, and new lesions appeared with each attempt to reduce the dose. Azathioprine $(100 \mathrm{mg} /$ day) and erythromycin $(2 \mathrm{~g} /$ day) were associated with the treatment, but both produced little response. The patient currently presents partial control of the disease, in use of prednisone (10mg/day) and dapsone (50mg/day).

\section{Case 2}

Male patient, 51 years of age, reported pruritic skin lesions in the previous month. Upon examination, he presented tense blisters throughout the tegument, some with a rosette configuration ( $\mathrm{Fi}$ gure 4). He reported no comorbidities or use of medications. With bullous pemphigoid and epidermolysis bullosa acquisita as the diagnostic hypotheses, biopsies were performed for anatomopathology and DIF, and patient was started on prednisone $[60 \mathrm{mg} /$ day $(0.6 \mathrm{mg} / \mathrm{kg})]$. One month later the patient showed significant improvement in the lesions. Biopsies revealed subepidermal bullous dermatosis with the presence of linear IgA fluorescence in the basement membrane zone - findings consistent with linear IgA dermatosis. Prednisone was reduced, and dapsone was added (100mg/day). Three weeks later, the patient returned with mild hemolysis, and dapsone was reduced to $50 \mathrm{mg} /$ day. Tapering of prednisone and

Received 04 June 2017

Accepted 10 August 2017.

* Work conducted at the Department of Dermatology, Universidade Federal de São Paulo (Unifesp), São Paulo (SP), Brasil.

Financial support: None.

Conflict of interest: None.

Department of Dermatology, Escola Paulista de Medicina, Universidade Federal de São Paulo (Unifesp), São Paulo (SP), Brasil.

Department of Pathology, Escola Paulista de Medicina, Universidade Federal de São Paulo (Unifesp), São Paulo (SP), Brasil.

MAILING ADDRESS:

Taila Yuri Siqueira Machado

E-mail: taila_ma@yahoo.com

(C2018 by Anais Brasileiros de Dermatologia 

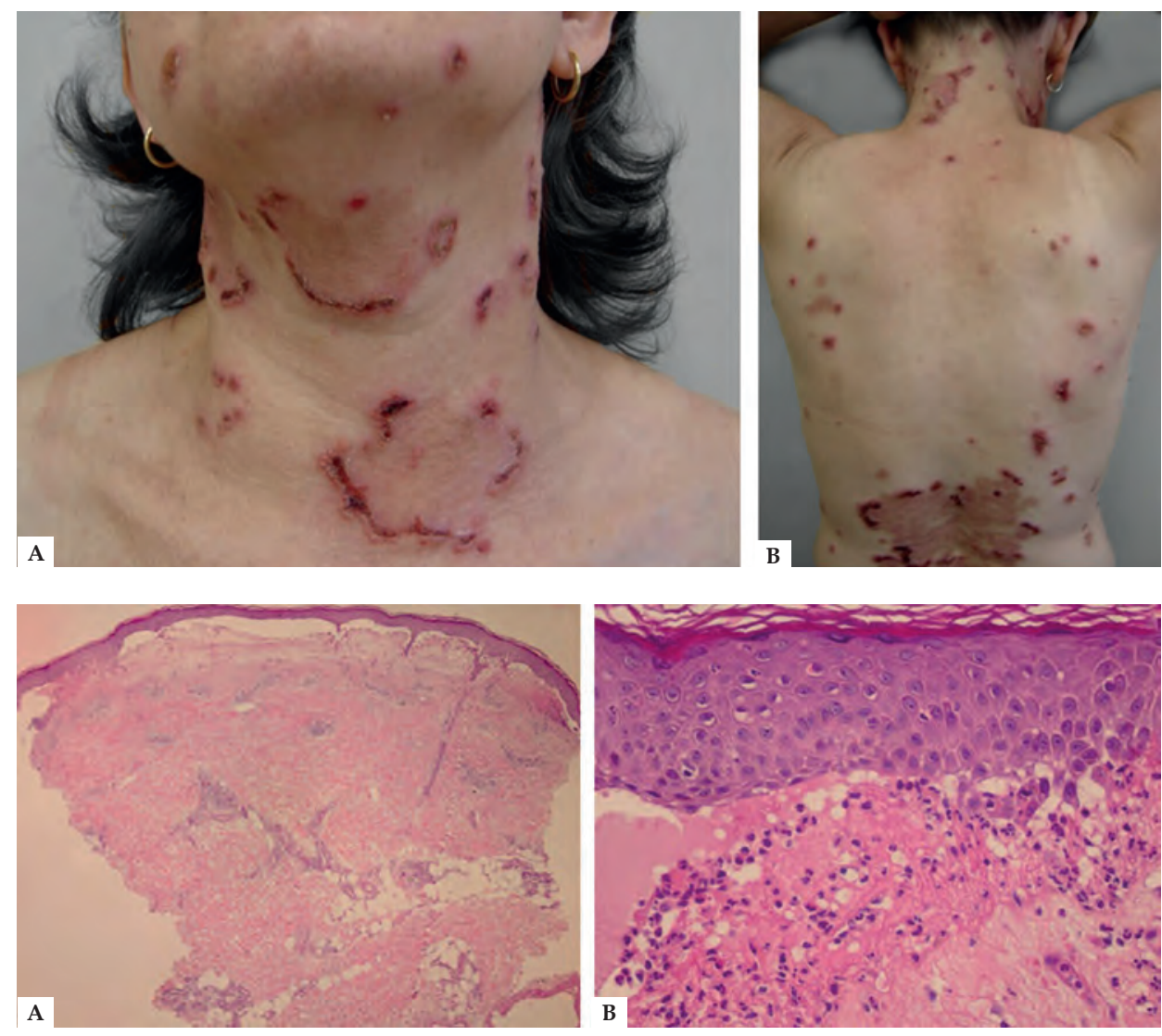

Figure 1: Vesicles and hemorrhagic crusts with an arciform configuration on the anterior cervical region (A) and posterior cervical region and on the back (B)

Figure 2: A - Subepidermal blister with serous content (Hematoxylin \& eosin, X40). B - Content of blister with predominance of neutrophils and serous material. Next to the cleavage zone there are microabscesses in the papillary dermis (Hematoxylin \& eosin, X400)

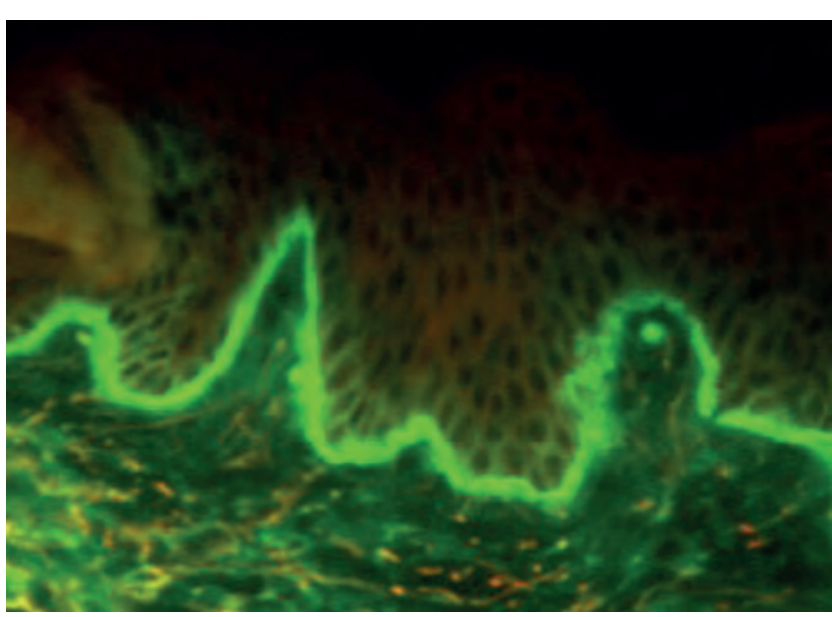

Figure 3: Linear IgA deposition in the basement membrane zone on direct immunofluorescence

dapsone was maintained. Two and a half years after onset of symptoms, patient had achieved complete control, and the medications were suspended.

\section{Case 3}

Female patient, 30 years of age, presented vesicles and blisters in the lumbar region, beginning one week previously. She was already on prednisone $(40 \mathrm{mg} /$ day), prescribed at another service, and brought her pathology results, consistent with bullous pemphigoid. As personal history, she reported polycystic ovaries and frequent use of analgesics for headache. Her condition worsened during tapering of prednisone, with erythematous lesions covered with pruritic vesicles and blisters located on the trunk and upper limbs, some with blisters on the periphery, giving a rosette appearance and suggesting linear IgA bullous dermatosis (Figure 5). Biopsies were performed for pathology and DIF, the results of which were, respectively, subepidermal bullous dermatosis with neutrophilic content and moderate linear IgA fluorescence in the basement membrane zone, confirming this diagnosis. Prednisone (20mg/ day) was prescribed. Three weeks later, dapsone $(100 \mathrm{mg} /$ day) was started, suspended two weeks later due to lack of response, besides symptomatic hemolysis. Patient's condition evolved with improvement after one month of prednisone, which the patient interrupted on her own. She returned one month later with relapse of the disease, and prednisone $(20 \mathrm{mg} /$ day) was prescribed again with monthly dose tapering until suspension.

\section{DISCUSSION}

Linear IgA bullous dermatosis is a rare autoimmune bullous disease with heterogeneous skin manifestations. There may be erythematous papules, urticaria-like plaques, or vesicobullous lesions. The latter may display an annular or arciform configuration or rosette appearance when new bullous lesions develop on the periphery of previous lesions. The disease can affect the flexor surfaces of the limbs and the trunk, as in bullous pemphigoid, or 


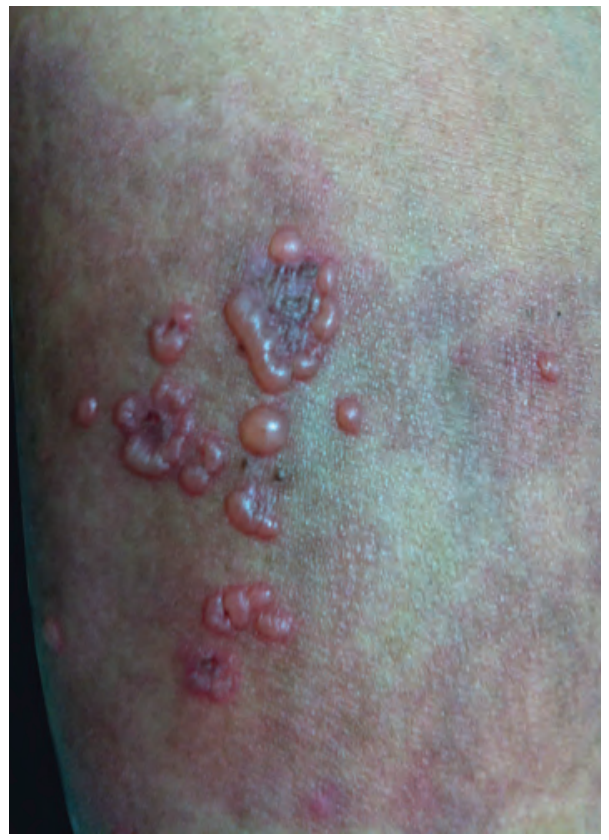

Figure 4: Tense vesicles and blisters with serous content on the back, with some on the periphery of previous lesions, setting up a rosette appearance the extensor aspects of the limbs, as in dermatitis herpetiformis; in addition, oral involvement occurs in the majority of cases. ${ }^{4}$ It may be spontaneous or drug-induced, with vancomycin reported most frequently. However, there have also been reports of its emergence after the use of amiodarone, nonsteroidal anti-inflammatory drugs, acetaminophen, and antibiotics other than vancomycin, such as ceftriaxone and penicillin. ${ }^{5}$

Diagnosis is based on three criteria: i) vesicles or blisters on the skin or mucosae; ii) subepiderrmal blisters with neutrophilic infiltrate on anatomopathology; and iii) IgA deposits on the basement membrane zone with a linear pattern and absence of other immunoglobulins on DIF. ${ }^{2}$

Dapsone is the drug of choice, as in dermatitis herpetiformis. Its side effects are hemolytic anemia, leucopenia, methemoglobinemia, and altered liver enzymes. Monotherapy with dapsone is

\section{REFERENCES}

1. Prystowsky S, Gilliam JN. Benign chronic bullous dermatosis of childhood. Linear IgA and C3 deposition on the basement membrane. Arch Dermatol. $1976 ; 112: 837-8$

2. Egan CA, Zone JJ. Linear IgA bullous dermatosis. Int J Dermatol. 1999;38:818-27.

3. Wojnarowska F, Marsden RA, Bhogal B, Black MM. Chronic bullous disease of childhood, childhood cicatricial pemphigoid, and linear IgA disease of adults. A comparative study demonstrating clinical and immunopathologic overlap. J Am Acad Dermatol. 1988;19:792-805.

4. Guide SV, Marinkovich MP. Linear IgA bullous dermatosis. Clin Dermatol. 2001;19:719-27.

5. Chanal J, Ingen-Housz-Oro S, Ortonne N, Duong TA, Thomas M, Valeyrie-Allanore L, et al. Linear IgA bullous dermatosis: comparison between the drug-induced and spontaneous forms. Br J Dermatol. 2013;169:1041-8.

$\begin{array}{ll}\text { Taila Yuri Siqueira Machado } & \text { (D) ORCID 0000-0002-5121-1858 } \\ \text { Milvia Maria Simões e Silva Enokihara } & \text { (D) ORCID 0000-0002-3340-4074 }\end{array}$
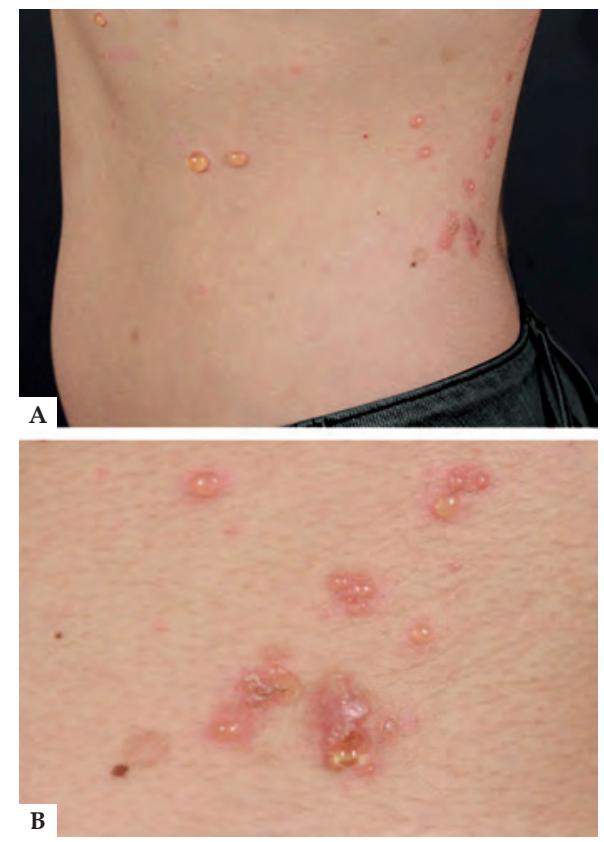

Figure 5: Lesions on the trunk: tense vesicles and bullae with serous content, some on an erythematous base (A); detail of lumbar region $(\mathbf{B})$

sufficient in many cases, but the association with corticosteroids and immunosuppressants such as azathioprine and cyclophosphamide may be necessary, especially due to dapsone's side effects, which limit its daily dose, as occurred in the three cases reported here. ${ }^{6,7}$

Some cases of therapeutic success have been reported with the association of tetracycline and nicotinamide or with macrolides, such as erythromycin, as occurs with bullous pemphigoid..$^{8,9}$

It is difficult to precisely define the duration of treatment, since most cases relapse during tapering of the medication, requiring a new increase. Mean treatment time of 4.6 years has been reported in cases of spontaneous linear IgA dermatosis. ${ }^{10}$

In conclusion, linear IgA dermatosis is a rare autoimmune bullous disease, frequently confused with other bullous diseases like bullous pemphigoid and Duhring's dermatitis herpetiformis, due to the clinical heterogeneity. Correct early diagnosis is essential to provide adequate treatment. $\square$

6. Zone JJ. Clinical spectrum, pathogenesis and treatment of linear IgA bullous dermatosis. J Dermatol. 2001;28:651-3.

7. Fortuna G, Marinkovich MP. Linear immunoglobulin A bullous dermatosis. Clin Dermatol. 2012;30:38-50.

8. Peoples D, Fivenson DP. Linear IgA bullous dermatosis: successful treatment with tetracycline and nicotinamide. J Am Acad Dermatol. 1992;26:498-9.

9. Cooper SM, Powell J, Wojnarowska F. Linear IgA disease: successful treatment with erythromycin. Clin Exp Dermatol. 2002;27:677-9.

10. Lings K, Bygum A. Linear IgA bullous dermatosis: a retrospective study of 23 patients in Denmark. Acta Derm Venereol. 2015;95:466-71.

$\begin{array}{ll}\text { Tatiana Miyuki lida } & \text { (D) ORCID 0000-0001-7097-006X } \\ \text { Adriana Maria Porro } & \text { (D) ORCID 0000-0003-0736-4790 }\end{array}$

How to cite this article: Machado TYS, Enokihara MMSS, Iida TM, Porro AM. Adult linear IgA bullous dermatosis: report of three cases. An Bras Dermatol. 2018;93(3):435-7. 